\section{Chronische Rückenschmerzen multimodal behandeln}

\begin{abstract}
Multimodale Therapie gilt als Schlüsselbegriff in der Behandlung chronischer Schmerzen. Wie sich das praktisch umsetzen lässt, berichtete eines der erfolgreichsten deutschen Versorgungsteams vom überregionalen Hamburger Rückenzentrum „Am Michel", aus ärztlicher, psychologischer und physiotherapeutischer Perspektive.
\end{abstract}

$E^{\mathrm{n}}$ ntscheidend ist der Einstieg mit einem interdisziplinären Diagnostiktag, bei dem ein Arzt, ein Psychologe und ein Physiotherapeut den Patienten nacheinander fachspezifisch und anschließend fachübergreifend beurteilen und prüfen, erläuterte der Orthopäde Dr. Joachim Mallwitz aus Hamburg: „Wir definieren für uns im Erstkontakt mit dem Patienten ein Therapieziel, um nicht ein unrealistisches Therapieziel entstehen zu lassen." Dabei durchläuft jeder Patient eine 45 - bis 60 -minütige Evaluation der drei beteiligten Disziplinen. Auf die ärztliche Anamnese und eingehende körperlicher Untersuchung inklusive der körperlichen Leistungsfähigkeit und Ermittlung der alltäglichen Beanspruchung folgt eine psychologische und psychosoziale Beurteilung mit Interview und fokussierten Fragebögen sowie eine funktionsorientierte physiotherapeutische Evaluation. Im Team wird dann für geeignete Patienten eine Therapieperspektive für eine mehrwöchige Behandlung in der Tagesklinik erarbeitet.

\section{Geeignete Patienten für die multimodale Therapie erkennen}

„Wir müssen einen chronischen Kreuzschmerzpatienten als Langzeitpatienten sehen“, so Mallwitz. Eine völlige Beschwerdefreiheit sei in den meisten Fällen ein unrealistisches Therapieziel. Entscheidend sei es, „Befunde der Bildgebung in den allermeisten Fällen zu relativieren" und möglichst eine Funktionsdiagnose zu stellen: „Die Diagnose eines Bandscheibenvorfalles hilft uns nicht weiter. Ab einem gewissen Alter gehört eine Protrusion zu uns wie graue Haare." Sie finde sich auch bei fast $90 \%$ aller untersuchten asymptomatischen Patienten. Nur funktionelle Diagnosen wie ein zervikales Dekonditionierungssyndrom bei funktioneller Instabilität der Bewegungssegmente, muskuläre Dysbalancen der Nacken-Hals-Muskulatur oder neuromeningeale Gleitstörungen seien aktiv behandelbar. Der frühere Physiotherapeut Mallwitz versteht seine jetzige Rolle als Arzt vor allem als Coach für das Team und für den Patienten. Früher galt, „wenn ein Patient Schmerz meldet, Stufenbettlagerung und Traktion - mehr nicht! Jetzt gemeinsam im Team den Umgang mit dem Schmerz zu entwickeln, ist der entscheidende Fortschritt.“

\section{Direktes Gespräch zur psychologischen Reflexion}

Im psychologischen Assessment stehen Schmerzsymptomatik, berufliche Anamnese, psychopathologische Befunde wie etwa Alkoholkonsum, aber auch der soziale Kontext des Patienten im Mittelpunkt, betonte Britta Maurus, DiplomPsychologin aus Hamburg. Typische FraSchmerz verändert, wie habe ich mich durch den Schmerz verändert und wird dieser durch psychische Faktoren beeinflusst? Herausgearbeitet werden aber auch Arbeitszufriedenheit, Betriebsklima, Arbeitsmotivation und die Reaktionen des Arbeitgebers auf die Erkrankung als wichtige Einflussfaktoren der Schmerzbewältigung. „Wir fragen in diesem ersten Gespräch ziemlich direkt", erläuterte die Psychologin. Sehr wichtig sei der soziale Kontext des Patienten, also seine Zufriedenheit mit Lebensbedingungen, Freundeskreis, Hobbies sowie beruflichem und familiärem, aber auch erweitertem sozialen Umfeld. Als Warnsignale (yellow flags) für eine Chronifizierung gelten ausgeprägtes Vermeidungsverhalten und Inaktivität, Forderung nach sozialer Unterstützung, ein ausgeprägtes Bedürfnis nach Ruhezeiten oder Bettruhe, aber auch ein extremes gen sind: Was hat sich durch den
Durchhalten oder die Negierung eigener Grenzen. Spezifische schmerzbezogene Daten des Patienten, etwa die Stimmungsreaktion bei Schmerz, der Grad der Beeinträchtigung bei der Arbeit und der Freizeit sowie der zusätzliche Einfluss von körperlichen und seelischen Belastungen werden ebenso erfragt.

\section{Durch Training zurück zur Alltagsbelastung}

Physiotherapeutische Schwerpunkte sind die Präferenz von Funktion gegenüber Struktur sowie das sukzessive Zurückführen des Patienten in die körperliche Belastung, sagte Michael Richter, Physiotherapeut des Hamburger Rückenzentrums. Was hat der Patient an Vortherapie, was ist sein funktionelles Hauptproblem, was behindert ihn im Alltag, wodurch kommt eine Linderung zustande? Hier gelte, sich nicht auf der biomedizinischen Strukturebene zu verlieren, auf der man - für Langzeit-Patienten üblich - stets muskuläre Verspannungen, Blockierungen und Dysfunktionen finde. Richter setzt auf physiotherapeutische Prä-Post-Tests, mit denen Fortschritte erkenn- und quantifizierbar werden. Als Beispiele nannte er Stehen über zehn Minuten oder die Belastbarkeit beim Heben, gemessen etwa mit dem PILE-Test. Wichtig für den möglichen Behandlungsfortschritt sei auch die Definition persönlicher Ziele durch den Patienten, etwa die Wiederaufnahme einer Sportart, aber auch eine normale körperliche Belastbarkeit in Arbeitssituationen. Für das Üben von Alltagsbelastungen gilt dabei das Motto, „trainiere das, was schwerfällt". Hilfsmittel sind ein Gerätebasiertes Training der Maximalkraft sowie der Wirbelsäulenstabilisation unter enger Rückkopplung mit dem Arzt.

Bei über $70 \%$ der Patienten mündet dieses initiale Assessment in positive Therapieempfehlungen für eine mehrwöchige tagesklinische Behandlung an fünf Tagen der Woche, resümierte Maurus. Ungeeignet seien allerdings Patienten, die planen, die Rente einzureichen oder bereits ein Rentenverfahren laufen haben.

Dr. Andreas Häckel

Lunchseminar „Interdisziplinäres Assessment des chronifizierten Kreuzschmerzpatienten" Frankfurt am Main, 3.3.2016 